\title{
Sozialräumliche Segregation und Bildungsungleichheiten in der Grundschule - eine Bestandsaufnahme
}

\author{
Ralf Parade $\cdot$ Friederike Heinzel
}

Online publiziert: 23. Juli 2020

(C) Der/die Autor(en) 2020

Zusammenfassung Während bundesweit das 100-jährige Bestehen der Grundschule zelebriert und dabei häufig an das Gründungsversprechen erinnert wird, es handele sich um eine „Schule für alle“, wird im Beitrag auf Basis eines Überblicks empirischer Studien zu sozialräumlicher Segregation in Deutschland und damit zusammenhängenden Bildungsungleichheiten in der Grundschule geprüft, inwiefern jenes Credo der aktuellen empirischen Befundlage standhält. In der Folge wird argumentiert, dass sich künftig die Grundschulpädagogik vermehrt der Problematik anzunehmen hat, um dafür Sorge zu tragen, dass sich die Grundschule dem annähert, was sie seit jeher zu sein beansprucht.

Schlüsselwörter Bildungsungleichheiten · Segregation · Sozialraum ·

Grundschule $\cdot$ Schulwahl

\section{R. Parade $(\varangle)$}

Institut für Erziehungswissenschaft, Universität Kassel, Gottschalkstraße 12, 34127 Kassel,

Deutschland

E-Mail: ralf.parade@uni-kassel.de

Prof. Dr. F. Heinzel

Institut für Erziehungswissenschaft, Universität Kassel, Nora-Platiel-Straße 1, 34127 Kassel, Deutschland

E-Mail: heinzel@uni-kassel.de 


\title{
Socio-spatial segregation and educational inequalities in primary school-a review
}

\begin{abstract}
The 100th anniversary of the primary school is celebrated nationwide. It was founded on the promise that it creates "a school for everybody". This article examines, if this credo can be confirmed based on an overview of empirical studies on socio-spatial segregation in Germany and related educational inequalities in primary schools. In consequence, we argue that future primary school pedagogy needs to attend to these issues in order to ensure that primary school comes close to what it was historically intended to be.
\end{abstract}

Keywords Educational Inequalities · Segregation - Social Space · Primary School School Choice

\section{Einleitung}

Mit Blick auf Bildungsbenachteiligung im deutschen Schulsystem wird für die vergangenen Dekaden ein Wandel von der Kunstfigur der ,,katholischen Arbeitertochter vom Lande“ hin zum ,muslimischen Migrantensohn in der Großstadt" konstatiert (vgl. Geißler 2005; Koch 2008, S. 581; Kahlert 2008, S. 788; Hopf 2017, S. 26). Unter den Analysekategorien, die in diesen statistischen Prototypen verdichtet wurden, stellt der Raum jenen Faktor dar, der seit der ersten „Bildungskatastrophe“ kontinuierlich in Vergessenheit geriet (vgl. Ditton 2007, S. $21 \mathrm{f}$.) und nun erst seit dem „PISA-Schock“ zunehmend (wieder) an Bedeutung im Sinne der Erforschung sozialer und ethnischer Segregation in Quartieren und Schulen gewinnt (vgl. etwa Ditton und Krüsken 2007; Baur 2013; Terpoorten 2014; Ditton 2014; Berkemeyer und Meißner 2017; Jurzczok 2019; Mayer und Koinzer 2019). Ein solcher „spatial turn“" verwundert wenig, vergegenwärtigt man sich die größer werdende Kluft zwischen sozialen Lagen in großstädtischen Quartieren. Im Zuge dessen wird häufig davon ausgegangen, ,dass benachteiligende oder privilegierende Faktoren des Sozialraumes vermittelt über die schulischen Lernkontexte selbst auf den Bildungserwerb von Kindern und Jugendlichen wirken und neben familialen Ressourcen und individuellen Merkmalen nachteilige oder günstige Konsequenzen zeitigen" (Jurczok und Lauterbach 2014, S. 136). Ditton und Krüsken (2007, S. 36) konstatierten dazu bereits vor über zehn Jahren, Sozialindizes städtischer Quartiere würden ,oft eine erstaunlich (erschreckend?) gute Prognose des zu erwartenden Lernerfolgs und der Bildungsteilhabe [erlauben, d.V.]“".

In diesem Sinne ist es naheliegend, den Zusammenhang von sozialräumlicher Segregation und Bildungsungleichheiten in der Grundschule einer eingehenden Prüfung zu unterziehen. Nach kurzen begrifflichen Annäherungen an den Gegenstand werden im Beitrag zunächst Tendenzen sozialräumlicher Segregation in Deutschland aufgezeigt (2). Im Anschluss rückt das Verhältnis von residentieller und schulischer Segregation in den Blick, wobei insbesondere Grundschulwahl und der expandierende Privatschulsektor einer gesonderten Betrachtung bedürfen (3). Nachfolgend werden Befunde zu Nachbarschafts- sowie Kompositionseffekten auf Schulleistun- 
gen referiert (4). Inwiefern sozialräumliche Kontexte und Klassenkomposition in der Grundschule mit dem Übertritt auf die weiterführende Schule in Zusammenhang stehen, wird in einem weiteren Gliederungspunkt behandelt (5). Eine kurze Zusammenfassung sowie ein Ausblick auf offene Forschungsfragen schließen den Beitrag (6).

\section{Sozialräumliche Segregation in Deutschland}

Da in diesem Beitrag von sozialräumlicher Segregation die Rede ist, sollte geklärt werden, worum es sich dabei überhaupt handelt. Was zunächst trivial erscheint, ist nach Betrachtung der nach jeweiligen Gegenstandsbezügen differierenden Verständnisse des Begriffes „Sozialraum“ bereits klärungsbedürftig. Wir begreifen „Sozialraum“ - Kessl und Reutlinger (2019, S. vii) folgend - ,als Raumbegriff, der davon ausgeht, dass Räume ständig (re)produzierte Gewebe sozialer Praktiken darstellen. Bewusst ist dabei von einem Gewebe die Rede, [...] denn in (Sozial)Räumen sind heterogene historische Entwicklungen (z. B. baulich-ästhetische Praktiken), kulturelle Prägungen (z. B. soziale Ordnungen) und politische Entscheidungen (z. B. raumplanerische Programme) eingeschrieben und bilden dabei zugleich einen relativ stabilen und damit das soziale Tun prägenden Verbund“. Kessl und Reutlinger verweisen hier auf ein heutzutage dominantes Raumverständnis, das frühere Essentialisierungen abstreift und stattdessen die soziale Konstruiertheit von Räumen sowie ihre Rückwirkung auf Menschen und ihr Handeln aus einer prozessualen Perspektive mitdenkt (vgl. Hummrich 2015; Wehrheim 2016). Segregation nun beschreibt die räumliche Ungleichverteilung verschiedener Bevölkerungsgruppen als Widerspiegelung sozialer Disparitäten, ethnischer Differenzierung oder altersspezifischer Wohnraumansprüche und -vorlieben (vgl. Farwick 2012). Insbesondere die ersten beiden Dimensionen können mit Blick auf Bildungsungleichheiten als relevant gelten, doch stellt sich zunächst die Frage nach ihren jeweiligen Ausprägungen. Friedrichs (2011, S. 58f.) stellte hier mit Blick auf die Nachwendezeit in Deutschland fest, dass zwar im Zeitraum von 1990 bis 2004 ethnische Segregation rückläufig war, jedoch soziale Segregation zugenommen hat. Aktuelle Zahlen bestätigen diesen Trend. So konnten Helbig und Jähnen unter Einbezug der Daten von 74 deutschen Städten zeigen, dass zwar die ethnische Segregation weiter gesunken ist, sich insgesamt die soziale Segregation im Zeitraum von 2005 bis 2014 jedoch um 10,5\% ausgeweitet hat, was Ostdeutschland mit 23,4\% in noch stärkerem Maße betrifft (vgl. Helbig und Jähnen 2018, S. 113). Außerdem konnte (mit Ausnahme einer Stadt) festgestellt werden, dass die soziale Segregation von Kindern (bzw. Haushalten mit Kindern) noch höher ist als die allgemeine soziale Segregation (vgl. Helbig und Jähnen 2018, S. 114). Demnach ,gibt es in 36 der betrachteten Städte Nachbarschaften, in denen der Anteil von Kindern in Haushalten mit SGB-II-Bezug bei über $50 \%$ liegt - also mehr als die Hälfte der Kinder im Quartier arm ist. In 15 deutschen Städten leben über 10\% aller Kinder in diesen von Armut geprägten Quartieren“ (Helbig und Jähnen 2018, S. 114). 


\section{Schulische Segregation, Grundschulwahl und Privatschulsektor}

Geht man davon aus, dass die Grundschule ihrem Selbstverständnis nach als „Schule für alle" eine Integrationsfunktion besitzt, kann ein Grouping der Schülerschaft nach Ethnie oder sozioökonomischem Status kaum intendiert sein. Nichtsdestotrotz resultiert eine solche schulische Segregation u. a. daraus, dass sich bestimmte soziale Gruppen in spezifischen Quartieren konzentrieren. Aus der Zuweisung der Grundschule im Einzugsgebiet gemäß dem Sprengelprinzip und einer damit verbundenen eingeschränkten Grundschulwahl ergibt sich alsdann, „,dass Schulen aus sozial schwachen Bezirken oder aus Bezirken mit einem hohen Anteil an Migranten diesbezüglich homogene Schul- und Klassenumwelten aufweisen“ (Bellin 2009, S. 72). Für Deutschland hat bereits eine Vielzahl an Studien auf eine derartige Homogenisierung der Schülerschaft in Relation zum sozialräumlichen Einzugsgebiet der Grundschule aufmerksam gemacht (vgl. z. B. Noreisch 2007; Fincke und Lange 2012), wodurch Ergebnisse einer PISA-Sonderauswertung konterkariert werden, nach denen gerade die soziale Mischung der Schulklasse resilienzverstärkend auf sozioökonomisch benachteiligte Schüler*innen wirkt (vgl. OECD 2018, S. 8).

Dennoch wäre es zu kurz gegriffen, anzunehmen, dass sich residentielle einfach in schulische Segregation übersetzt. Das könnte zudem nicht erklären, warum Letztere in verschiedensten europäischen Großstädten stets stärker ausgeprägt ist als die Segregation in der Nachbarschaft (vgl. Boterman et al. 2019, S. 3070). Vielmehr gründet schulische Segregation - wie Radtke bemerkt - auf dem komplexen ,Zusammenwirken der Faktoren Stadtentwicklung, Schulentwicklungsplanung, Schulprofilbildung, Übergangsentscheidungen bzw. -empfehlungen der Schulen und Wahlverhalten der Eltern" (Radtke 2007, S. 202). Mayer und Koinzer (2019, S. 269) haben hier kürzlich ein holistisches Modell vorgestellt, das das wechselseitige $\mathrm{Zu}$ sammenwirken von Bildungspolitik und -verwaltung, räumlicher Einbettung, Nachbarschaft, Einzelschule sowie elterlicher/familialer Schulwahl in seiner Komplexität abzubilden vermag.

Der letztgenannte Punkt, die elterliche Schulwahl, spielt im internationalen Diskurs bereits seit längerer Zeit eine tragende Rolle bei der Erforschung von schulbezogenen Ungleichheiten (vgl. etwa Ball 1993, 2003; Ball et al. 1996; aktuell: Berends et al. 2019; Rohde et al. 2019). Im Zuge der „Vermarktlichung“ des Schulsektors und einer Pluralisierung von Schulprofilen im Wettstreit um die jeweilige Klientel (vgl. Altrichter et al. 2011b) - auch im Primarbereich (vgl. Krüger et al. 2015, S. 44) - rückt das Thema zunehmend auch in Deutschland in den Fokus des Interesses. Denn gehäuft wird durch neue Angebotsstrukturen im Zusammenspiel mit elterlichen Bildungsstrategien das Sprengelprinzip ausgehebelt: „Eltern wählen Privatschulen an, stellen selten bewilligte Gastschulanträge, wechseln den Wohnort oder fingieren Umzüge, um ihrem Nachwuchs einen Platz an einer präferierten Grundschule zu sichern" (Bloch et al. 2019, S. 270). Bereits 2012 konstatierten Fincke und Lange (2012, S. 2), dass ,,[r]und 10\% der Eltern in Großstädten für ihre Kinder einen Antrag auf Wechsel der behördlich zugewiesenen Grundschule [stellen]“ und insbesondere sogenannte „bildungsnahe“ Elternhäuser sind es, die zusehends aus „prekären“ Quartieren abwandern (vgl. Baur 2013, S. 11), was einer Homogenisierung der Stadtteile Vortrieb leistet. Auf das Schulwahlverhalten 
jener Klientel ist es auch primär zurückzuführen, dass sich für den Privatschulsektor - trotz insgesamt sinkender Schüler*innenzahlen - eine anhaltende Expansion verzeichnen lässt (vgl. zur Übersicht Koinzer und Leschinsky 2009; Ullrich und Strunck 2012) und die Anteile der Schüler*innen an privaten Grundschulen stetig gestiegen sind (vgl. Stirner et al. 2019, S. 442). Wirft man nun einen Blick auf elterliche Selbstpositionierungen, so kommt die Hallenser Forscher*innengruppe um Breidenstein zu dem interessanten Ergebnis, dass in ihrem Sample schulwahlaktive Eltern Segregation ausdrücklich nicht goutieren, sondern sich auf Ideale der Egalität und Chancengleichheit berufen. Demgegenüber jedoch gilt gleichfalls die Orientierung an der Verantwortung für das eigene Kind. Aktive Schulwahl wird so zum Ausdruck guter Elternschaft und legitimiert damit letztlich auch schulische Segregation (vgl. Roch et al. 2019, S. 117; Breidenstein et al. 2020, S. 52 ff.).

Hinsichtlich der Erklärung tiefer liegender Motive und Ursachen elterlicher Schulwahl lassen sich zwei in der bildungsbezogenen Ungleichheitsforschung häufig anzutreffende Zugänge unterscheiden. Zum einen werden in Anlehnung an sekundäre Herkunftseffekte bei Boudon (1974) Schulwahlentscheidungen als auf rationalen Kosten-Nutzen-Abwägungen (Erfolgsaussichten, Schulgeld, Fahrtweg usw.) gründend entworfen; zum anderen wird in Anlehnung an den kulturtheoretischen Ansatz Bourdieus (vgl. Bourdieu und Passeron 1971, 1973) Schulwahl mit dem Passungsverhältnis von familialem Habitus und sekundärem Habitus der Schule in Verbindung gebracht (vgl. ausführlich zum Theorem der kulturellen Passung Kramer 2011; Helsper et al. 2014).

Im Allgemeinen kann angenommen werden, dass Eltern an einem bestmöglichen Entwicklungs- und Lernumfeld für ihre Kinder gelegen ist, wodurch die (vermutete) Schulqualität, die ethnische sowie sozioökonomische Zusammenstellung der Schülerschaft und die fortgesetzte soziale Einbindung in das Wohnumfeld bei der Auswahl der Grundschule mit einfließen (vgl. Riedel et al. 2010; Schuchart et al. 2011). Hierbei zeigen sich jedoch Unterschiede in der Form, dass Eltern mit geringerem sozialen Status oder Migrationshintergrund tendenziell eher nach dem Kriterium der Standortnähe auswählen - etwa um Transportkosten gering zu halten oder um ein zum eigenen Hintergrund passförmiges Sozialisationssetting aufrechtzuerhalten und weniger nach der Zusammensetzung der Schülerschaft oder der vermeintlichen Schulqualität. Gegenläufige Tendenzen zeigen sich bei statushöheren Elterngruppen bzw. Elterngruppen ohne Migrationshintergrund (vgl. Riedel et al. 2010, S. 110f., 116). Etwa konnten Fincke und Lange (2012, S. 6, 9) in einer Berliner Fallstudie aufzeigen, dass jene Eltern ihre Wahl insbesondere mit dem Ziel einer Vermeidung des Besuchs von Schulen mit hohem Migrantenanteil treffen, was zu einer stärkeren ethnischen Segregation der Grundschulen führt, als dies ihre jeweiligen Schulbezirke vermuten ließen. „Da die tatsächliche Qualität einer Schule häufig nicht in Erfahrung gebracht werden kann, nehmen viele Eltern den Zuwandereranteil einer Schule als Indiz für das Lernumfeld und das Leistungsniveau“ (Fincke und Lange 2012, S. 12). Aus einer Analyse der Datensätze von IGLU und TIMSS geht hervor, dass $18 \%$ aller Grundschüler*innen in Deutschland eine Schule besuchen, in der über $50 \%$ der Schülerschaft einen Migrationshintergrund aufweisen ${ }^{1}$ - wobei 41,3\% der

\footnotetext{
1 folgend als ,segregierte Schule“ bezeichnet.
} 
Kinder mit Migrationshintergrund an einer solchen Schule lernen und nur 7,7\% der Kinder mit deutschen Wurzeln. Gleichzeitig besuchen 14,7\% der letztgenannten Gruppe eine Schule, in der kein einziges Kind mit Migrationshintergrund lernt. In großstädtischen Regionen vergrößert sich dieser Gegensatz noch einmal: Dort lernen $70 \%$ der Kinder mit einem Migrationshintergrund an einer Grundschule, in der über die Hälfte der Schülerschaft einen solchen Hintergrund besitzt, aber nur $17,1 \%$ der Schülerinnen und Schüler ohne Migrationshintergrund. Insgesamt gehen $4 \%$ aller Kinder in Deutschland in eine segregierte Grundschule, in der über die Hälfte der Schülerschaft armutsgefährdet ist, deren Familien also weniger als $60 \%$ des Nettoäquivalenzeinkommens zum Leben zur Verfügung haben. $9 \%$ der Kinder mit einem Migrationshintergrund besuchen eine segregierte Grundschule mit einem solchen Armutsanteil, jedoch nur unter 1\% der Kinder ohne Migrationshintergrund (vgl. Morris-Lange et al. 2013, S. 7 ff.).

Mit Blick auf eine Passung von elterlichen Präferenzen, kindlichen Fähigkeiten sowie schulischen Angebots- und Anforderungsstrukturen ist es nicht nur so, dass Eltern spezifisch profilierte öffentliche oder private Schulen anwählen, um diese zu gewährleisten und/oder Distinktionsgewinne zu verbuchen (vgl. Mayer 2017; Habeck et al. 2017; Schwarz et al. 2018), sondern es findet zuweilen - so es sich die Schulen aufgrund der Nachfrage leisten können - eine institutionenseitige Auswahl der passförmigen Klientel statt (vgl. Stošić 2015, S. 35; Krüger 2020). Das geschieht bei einigen Privatschulen bereits vermittels der Festlegung von Schulgeldzahlungen, die Artikel 7 Abs. 4 des Grundgesetzes zuwiderlaufen (vgl. Helbig und Jähnen 2018, S. 115).

Bis hierhin lässt sich sagen, dass die segregierende Wirkung von Schulwahl im wissenschaftlichen Diskurs kontrovers diskutiert wird. Sehen die einen jene als erwiesen an, deuten andere Befunde Gegenteiliges an: Bspw. zeigte sich in Linz nach der Implementierung freier Schulwahl kein signifikanter Anstieg der Segregation und auch keine Veränderung der sozialen Komposition der Schulen (vgl. Altrichter et al. 2011a, S. 237). Nach der Abschaffung der Sprengelpflicht in Nordrhein-Westfalen im Schuljahr 2008/2009 fanden Makles und Schneider (2012) ebenso keinen Zusammenhang zwischen den neuen Wahlmöglichkeiten und der Zusammensetzung der Schülerschaft. Auch bei einer kürzlich veröffentlichten Studie auf Basis der Bremer Schülerindividualstatistik konnte residentielle Segregation als größter Einflussfaktor auf die Zusammensetzung ausgemacht werden, wohingegen aktive Schulwahl keine bedeutenden Effekte zeitigte (vgl. Makles et al. 2019, S. 192).

\section{Nachbarschaftseffekte und schulische Kompositionseffekte auf Bildungserfolg in Grundschulklassen}

Fragt man nun danach, inwiefern sozialräumliche Segregation und Bildungsungleichheiten im Zusammenhang stehen, lassen sich verschiedene Linien verfolgen: Zunächst sind es Nachbarschaftseffekte bzw. Kontexteffekte des Wohnquartiers, die Einfluss auf den Bildungserfolg von Schüler*innen nehmen können. So generiert die jeweilige Konzentration einer bestimmten sozialstrukturell geprägten Anwohnerschaft mit ihren spezifischen Verhaltensweisen Milieus, die wiederum ihren Nie- 
derschlag in Orientierungsmustern der jeweiligen Anwohnerschaft finden und sich damit ermöglichend oder begrenzend auf Lebenschancen auswirken (vgl. Häußermann und Kronauer 2009, S. 158). Oder mit Bourdieu gesprochen: „Die im physischen Raum objektivierten großen sozialen Gegensätze [...] tendieren dazu, sich im Denken und Reden in Gestalt konstitutiver Opposition von Wahrnehmungs- und Unterscheidungsprinzipien niederzuschlagen, also selbst zu Kategorien der Wahrnehmung und Bewertung bzw. zu kognitiven Strukturen zu gerinnen“ (Bourdieu 2010, S. 119). Die angenommenen Wirkmechanismen der jeweiligen Wohnumgebung sind dabei vielgestaltig: Zum einen erfahren die Bewohner*innen über Sozialisationsprozesse eine bestimmte kulturelle Prägung - bspw. durch die Übernahme lokal dominanter Werte, Normen und Lebensentwürfe -, die auch epidemisch auf jene wirken kann, die neu hinzugezogen sind und Gruppenzugehörigkeit anstreben. Des Weiteren können sich materielle Gegebenheiten - etwa in Form begrenzter infrastruktureller Anbindung oder Konsummöglichkeiten - sowie mit der Umgebung verbundene Fremdzuschreibungen im Sinne von Stigmatisierungen limitierend auf Handlungs- und Entwicklungsmöglichkeiten auswirken (vgl. Häußermann und Kronauer 2009, S. 164; Ditton 2013, S. 178).

Doch auch wenn eine (un)mittelbare Einflussnahme des Sozialraums einleuchtend erscheint, gestaltet sich die empirische Analyse von Nachbarschaftseffekten auf den Bildungserfolg schwierig - sowohl aufgrund der Mannigfaltigkeit als auch der wechselseitigen Beeinflussung verschiedener Faktoren (vgl. Nonnenmacher 2015; Horr 2016). So kann auch die Schule auf die Nachbarschaft zurückwirken, wenn vermittels hoher Schulqualität im Quartier die Immobilienpreise steigen und sich damit die Zusammensetzung der Nachbarschaft ändert (vgl. Mayer und Koinzer 2019, S. 272). Zwar konnte bspw. Helbig (2010, S. 676) mit Daten der Berliner ELEMENT-Studie für Grundschulen zeigen, dass ,die Sozialstruktur der umgebenden Nachbarschaft die Lese- und Mathematikkompetenzen von Schülern beeinflusst", doch wird zumeist angenommen, dass Nachbarschaftseffekte über Kompositionseffekte im Sinne der Zusammensetzung der Schülerschaft vermittelt sind (vgl. Horr 2016, S. 427).

Eine Vielzahl von Untersuchungen konnte bereits Klarheit darüber verschaffen, dass sich ein Teil der Leistungsvarianz in (Grund-)Schulklassen über solche Kompositionseffekte erklären lässt (vgl. zur Übersicht internationaler Studien Van Ewijk und Sleegers 2010; Dumont et al. 2013; zu Grundschulen siehe Bellin 2009, S. 77-82), wobei jedoch die Befunde zum Ausmaß inkonsistent und auch hier die Vermittlungswege noch lückenhaft erforscht sind. Festzuhalten bleibt jedoch, dass sich ,,[f]ür die Auswirkungen sozialer Kompositionsfaktoren [...] ein Einfluss auf die schulischen Leistungen sowohl für den sozioökonomischen Status als auch für die ethnische Zusammensetzung an Schulen [zeigt, d.V.]“ (Bellin 2009, S. 89). Die Zusammensetzung der Klasse kann u. U. im Sinne eines institutionellen Matthäuseffektes lernförderlich oder auch -hinderlich wirken und hat damit besondere Relevanz für den Bildungserfolg von Schüler*innen (vgl. Ditton 2013, S. 188, 190). Zudem kommen im Bericht der IGLU-Studie 2016 die Autor*innen nach Sichtung diverser Studien zu Kompositionseffekten zu dem Ergebnis, dass die Zusammensetzung der Klasse nicht bloß Einfluss auf individuelle Schulleistung und Übergangsentscheidun- 
gen, sondern auch im Sinne des Big-Fish-Little-Pond-Effekts auf Selbstkonzept und Lernmotivation der Schüler*innen nimmt (vgl. Bremerich-Vos et al. 2017, S. 301).

Kürzlich haben Helbig und Nikolai (2019) auf einen weiteren Umstand hingewiesen, den es mit Blick auf die soziale Lage der Schulen zu berücksichtigen gilt. Auf Basis der amtlichen Schulstatistik konnten die Autoren für Berlin herausarbeiten, dass eine niedrigere Unterrichtsabdeckung in Grundschulen mit einem erhöhten Anteil an sozial schwachen Kindern in Zusammenhang gebracht werden kann. Ebenso geht mit einer solchen Zusammensetzung der Schülerschaft eine erhöhte Anzahl an Vertretungsstunden sowie ein größerer Anteil an Quereinsteiger*innen unter den Lehrpersonen einher. Außerdem zeigte sich, dass sozial privilegierte Schulen bei Schulinspektionen mit Blick auf Schulqualität besser bewertet werden (vgl. ebd., S. 13-24). Helbig und Nikolai leiten aus diesen Befunden den Vorschlag einer sozialindexgesteuerten Ressourcenzuweisung ab, durch die Schulen, in den sich sozial schwächere Schüler*innen konzentrieren, eine bessere pädagogische Förderung ermöglichen könnten. Die Verbindung zwischen sozialpolitischen Problemen und pädagogischen Handlungsmöglichkeiten deutet sich hier an.

\section{5 Übergänge in weiterführende Schulen}

Da der Übergang in die weiterführende Schule als einer ,,der bedeutendsten Übergänge in der Bildungsbiografie eines Heranwachsenden“ (Baumert et al. 2010, S. 5) gilt, konzentrieren sich auch viele Studien zu räumlichen Bildungsdisparitäten auf diese Schwelle.

Ditton konnte im Rahmen der KOALA-S-Studie zeigen, dass Übergangsempfehlungen der Lehrkräfte, elterliche Bildungsaspirationen sowie die Anmeldequoten an weiterführenden Schulen im Verhältnis zur Region stark variieren (vgl. Ditton 2014, S. 24). „Die Bildungsaspirationen, Übertrittsempfehlungen und Schulanmeldungen stehen in einer signifikanten Beziehung zur sozialen Zusammensetzung der Schulklassen. Mit ansteigendem sozialem Status der Schulklasse (ISEI, EGP), höherem mittleren Sozialprestige (SIOPS) und höherem mittleren Bildungsniveau ergeben sich jeweils ansteigende Aspirationen, Empfehlungen und Übertritte“ (ebd.).

Mit Blick auf Gymnasialempfehlungen zeigen Untersuchungen mehrheitlich soziale Kompositionseffekte dergestalt, dass sich die Wahrscheinlichkeit einer Gymnasialempfehlung mit steigendem durchschnittlichen Sozialstatus einer Klasse oder Schule vergrößert (vgl. zur Übersicht Dumont et al. 2014, S. 153). Neumann et al. (2010, S. 248) konnten zeigen, dass sich ein steigender Anteil der Eltern mit Abitur in einer Klasse begünstigend auf den Übergang in das Gymnasium auswirkt, wobei sich der Kompositionseffekt einerseits auf den elterlichen Schulwunsch, aber auch auf die Schulempfehlung der Lehrkräfte auszuwirken scheint. Hinsichtlich der Wahlfreiheit der Eltern beim Übergang in die weiterführende Schule ergibt sich ähnlich wie mit Blick auf die Auswahl der Grundschule verhandelt wird - die Bewegung hin zu einer zusätzlichen „Entmischung“ der Schülerschaft, da Eltern mit höherem sozioökonomischen Status sozial selektive Übergangsentscheidungen gemäß den eigenen Bildungsaspirationen (zu) fällen (vermögen) (vgl. Dumont et al. 2014, S. 153). Eine ähnliche Tendenz sieht auch Clausen im Zuge der Ergebnisse 
einer an Mannheimer Grundschulen durchgeführten Untersuchung zur schicht- und bildungsabhängigen Wahl der weiterführenden Schule. Es sei ,zu fragen, inwieweit die Schullandschaft in der Zusammensetzung der verschiedenen Schülerschaften die sozialräumliche Ungleichheit einer Gesellschaft abbildet oder nicht sogar durch Entmischungs- und Klumpungseffekte stärker segregiert ist als die Welt um sie herum. Sofern eine Erweiterung der Wahlfreiheit nicht von Maßnahmen begleitet wird, die auch bildungsferneren Schichten eine elaboriertere Entscheidung ermöglichen, so ist zu erwarten, dass diese Freiheit primär den Kundigeren und Erfahrenen zugute kommt“ (Clausen 2006, S. 87). Jene „Kundigeren“ und „Erfahreneren“ sind zumeist - auch aufgrund der Zusammenstellung der Klientel der jeweiligen Schule - sozial vernetzt. Lintorf et al. (2017) merken hierzu unter Berücksichtigung verschiedener Studien an, dass Eltern die Entscheidungen zur Auswahl der weiterführenden Schule in einem sozialen Kontext treffen und sich ggf. an den Normen und Idealen ihrer sozialen Netzwerke orientieren. Insbesondere aber seien informative Einflüsse anderer Eltern ausschlaggebend für die Übergangsentscheidung (vgl. Lintorf et al. 2017, S. 714 ff.). Unter Verwendung des TIMSS-IGLU-2011-Datensatzes konnten die Autorinnen die Ergebnisse anderer Studien zu Effekten der sozialen Komposition auf die Schulwahl replizieren, da sich, ,in Klassen mit einem höheren mittleren sozioökonomischen Status eine höhere individuelle Wahrscheinlichkeit, nach der Grundschule auf das Gymnasium zu wechseln“ (Lintorf et al. 2017, S. 722f.), ergab. Des Weiteren wies die Studie gemäß dem postulierten Wirkungszusammenhang nach, dass ,ein unter der Klassenelternschaft geteilter Optimismus bezüglich der Erfolgschancen am Gymnasium in einem positiven Zusammenhang mit der individuellen Übergangsbereitschaft ans Gymnasium“ (Lintorf et al. 2017, S. 723) stand.

In einer groß angelegten Erhebung zu „Segregation und Bildungsdisparitäten am Übergang in die weiterführenden Schulen im Agglomerationsraum Ruhrgebiet“" untersuchte Terpoorten (2014) die Sozialstruktur von 14 Städten und setzte diese zu Daten der Schulstatistik hinsichtlich schulischer Übergänge sowie zu Geometriedaten in Verbindung. Bisherige Ergebnisse der regionalen Bildungsforschung wurden bestätigt: „Bildungschancen sind ungleich in den Städten verteilt und verlaufen entlang der Grenzen sozialer und sozialräumlicher Ungleichheit. In den Städten des Ruhrgebiets spiegelt sich die soziale und ethnische Segregation der Bevölkerung in einer räumlichen Bildungsdisparität. In den bürgerlichen Stadtgebieten besuchen die Kinder zumeist das Gymnasium - in sozial benachteiligten Gebieten werden verstärkt Gesamt- und Hauptschulen angewählt“ (Terpoorten 2014, S. 269). Schräpler und Weishaupt (2019, S. 435) aktualisierten vor Kurzem diesen Befund und stellten abermals für Nordrhein-Westfalen fest, ,dass es zwischen dem Sozialraum der Grundschulen und den Übergangsquoten zum Gymnasium einen starken Zusammenhang gibt".

Hauf (2007, S. 304) wies bereits vor einigen Jahren auf eine „Versäulung“ von bestimmten Grundschulen mit weiterführenden Schulformen hin, die in Verbindung zur Sozialstruktur der jeweiligen städtischen Quartiere steht, in denen diese Schulen gelegen sind. Weiterhin, so stellt der Forscher im Zuge seiner Analysen für den Raum Mannheim/Heidelberg von 1980-2002 fest, zeigt sich eine Persistenz innerstädtischer Bildungsdisparitäten - wenngleich auch Dynamiken der Bildungsbeteiligung 
feststellbar sind (vgl. Hauf 2007, S. 307 ff., 311). Letztlich kommen auch Jurczok und Lauterbach (2014, S. 152) im Fazit ihrer Forschung zu Übergängen an Berliner Grundschulen zu einem Schluss, der bereits im Zusammenhang mit der Wahl der Grundschulen angeklungen ist, nämlich, dass ,,bildungsnahe“ Familien Schulen in pivilegierten Bezirken bevorzugen, was dazu führt ,dass Schulen in benachteiligten Gebieten über die residentielle Segregation hinaus einer sozialen Verarmung der Schülerschaft unterliegen. Für den Stadtteil entsteht so eine doppelte Benachteiligung: Bildungsaffine Familien verlassen entweder mit der Einschulung ihrer Kinder den benachteiligten Bezirk oder sie senden beim Übergang in die weiterführende Schule ihre Kinder auf eine weit entfernte Schule, von der sie annehmen, dass sie besser ist."

In der Folge neuer Regionalisierungs- und Monitoringbestrebungen im Bildungswesen können mittlerweile kommunale bzw. regionale Bildungsberichte zu über 40 Regionen/Kreisen/Gemeinden eingesehen werden, die durch das BMBF-geförderte Projekt „Lernen vor Ort“" veranlasst wurden (vgl. Arbeitsgruppe „Lernen vor Ort“ 2016) und in denen bspw. die Sozialindizes der verschiedenen Schulsprengel, die Übergangsquoten an das Gymnasium in den jeweiligen Sprengeln sowie die Sprengel mit ethnisch segregierten Schulen sowie ihre Übergangsquoten transparent gemacht werden. Auch wenn im Grundschulsektor (noch) keine Leistungsrankings vorhanden sind, die die Grundschulwahl entsprechender Elternkreise u. U. beeinflussen könnten, können doch ,,[d]ie Übergangsquoten von Grundschulen auf das Gymnasium, die entweder schulisch kommuniziert werden oder aber als Teil des Rufs von Grundschulen kursieren [...] als leistungsbezogene Indikatoren für vertikale Differenzierung fungieren“ (Helsper et al. 2019, S. 256f.) und damit letztlich auch für elterliche Schulwahl relevant werden. Die Transparenzmachung von Übergangsquoten im Sinne von Bildungsmonitorings wäre hier eine weitere Orientierungshilfe für Eltern, die diese Informationen zu deuten und für Bildungsentscheidungen zu nutzen wissen.

\section{Fazit}

Vor Kurzem hielt der deutsche Bundespräsident, Frank-Walter Steinmeier, anlässlich des Festaktes „100 Jahre Grundschule“ zur Eröffnung des Bundesgrundschulkongresses eine Rede, die ambivalent anmutete: Einerseits, so Steinmeier, zähle die Grundschule ,zu den großen demokratischen Errungenschaften des Jahres 1919 [...]“, stehe „für den Aufbruch in eine gerechtere Gesellschaft“" (Steinmeier 2019, S. 2) und habe ,in der Bundesrepublik zu einer wirklichen Erfolgsgeschichte werden“ können (Steinmeier 2019, S. 3). Andererseits jedoch, so der Politiker in Anspielung auf aktuelle Segregationstendenzen und ihre Auswirkungen weiter, sei die Grundschule ,[a]n manchen Orten in unserem Land [...] eben jetzt schon keine für alle gemeinsame Schule mehr“ (Steinmeier 2019, S. 5).

In jener Ambivalenz, zum einen den nie realisierten Gründungsmythos der Grundschule, ,gemeinsame Schule für alle“ zu sein, heraufzubeschwören und die damit verbundenen Ideale zu tradieren, während zum anderen Befunde zu bildungsbezogenen Ungleichheiten Gegenteiliges nahelegen, scheint auch die Grundschulpädagogik 
bis heute verhaftet zu sein. Wie die Autor*innen zu zeigen versuchten, sieht sich die Grundschule vor allem im Kontext sozialräumlicher Segregation mit verschiedentlichen Problemen konfrontiert, die den Status als „Schule für alle“ prekär erscheinen lassen (vgl. dazu bereits Heinzel und Parade 2020). Hierbei handelt es sich nicht nur um ein sozialpolitisches Problem, das soziologischer und erziehungswissenschaftlicher Analysen bedarf, sondern es leiten sich daraus auch grundschulpädagogische Fragestellungen und Aufgaben ab.

Es wäre bspw. danach zu fragen, inwiefern die Grundschulpädagogik selbst am Mythos der Grundschule als „Schule für alle“ mitgewirkt hat und mitwirkt, obwohl doch - ähnlich dem meritokratischen Credo (vgl. etwa Vester 2006; Becker und Hadjar 2011) - längst klar zu sein scheint, dass sich Glaubenssätze nicht zwingend mit den empirisch auffindbaren Gegebenheiten decken oder gar dafür sorgen, dass diese verklärt werden. Warum des Weiteren die Grundschulpädagogik zu weiten Teilen ihre Zuständigkeit für die Erforschung des hier umrissenen Themenfeldes nicht anerkennt, sondern sich hauptsächlich Soziologie, Bildungsökonomie, Sozialgeographie und andere Fachrichtungen daran abarbeiten.

Als zukünftige Aufgabe kann außerdem formuliert werden, sich die Forderung nach intensiverer Förderung von Kindern an Grundschulen, in denen sich sozial benachteiligte Schüler*innen konzentrieren, vermehrt zu eigen $\mathrm{zu}$ machen und dafür passende Unterrichtskonzepte zu entwickeln und zu untersuchen. Diesbezüglich gilt es jedoch auch, Schulen mit einer bestimmten Zusammensetzung nicht als „Problemschulen“ zu adressieren und damit Negativzuschreibungen zu reifizieren. Ebenso ist eine Lehrer*innenbildung für die Grundschule vonnöten, die verstärkt auf Bildung im Bereich sozialer Inklusion zielt. Das schließt die Untersuchung von wie auch die Arbeit an Haltungen von Grundschullehramtsstudierenden mit ein. Auswirkungen der sozialräumlichen Segregation müssten im Zusammenhang mit den institutionellen Bedingungen der Grundschule, mit dem spezifischen pädagogischen und didaktisch-methodischen Handlungsfeld der Primarstufe und in Bezug auf die Bildungsprogramme für die Grundschule untersucht werden. Interessant wären etwa stadteilbezogene Vergleiche schulspezifischer Curricula.

Seine Rede auf dem Bundesgrundschulkongress schloss der Bundespräsident mit einer Aufforderung an die Zuhörerschaft: „Lassen Sie uns gemeinsam anpacken, damit diese Institution Grundschule auch in Zukunft bleiben kann, was sie 1919 noch werden sollte: eine Schule der Demokratie“ (Steinmeier 2019, S. 7). Vielleicht - so könnte angemerkt werden - müsste es auch „werden kann“ heißen.

Funding Open Access funding provided by Projekt DEAL.

Open Access Dieser Artikel wird unter der Creative Commons Namensnennung 4.0 International Lizenz veröffentlicht, welche die Nutzung, Vervielfältigung, Bearbeitung, Verbreitung und Wiedergabe in jeglichem Medium und Format erlaubt, sofern Sie den/die ursprünglichen Autor(en) und die Quelle ordnungsgemäß nennen, einen Link zur Creative Commons Lizenz beifügen und angeben, ob Änderungen vorgenommen wurden.

Die in diesem Artikel enthaltenen Bilder und sonstiges Drittmaterial unterliegen ebenfalls der genannten Creative Commons Lizenz, sofern sich aus der Abbildungslegende nichts anderes ergibt. Sofern das betreffende Material nicht unter der genannten Creative Commons Lizenz steht und die betreffende Handlung nicht nach gesetzlichen Vorschriften erlaubt ist, ist für die oben aufgeführten Weiterverwendungen des Materials die Einwilligung des jeweiligen Rechteinhabers einzuholen. 
Weitere Details zur Lizenz entnehmen Sie bitte der Lizenzinformation auf http://creativecommons.org/ licenses/by/4.0/deed.de.

\section{Literatur}

Altrichter, H., Bacher, J., Beham, M., Nagy, G., \& Wetzelhütter, D. (2011a). The effects of a free school choice policy on parents' school choice behaviour. Studies in Educational Evaluation, 37, 230-238.

Altrichter, H., Heinrich, M., \& Soukup-Altrichter, K. (2011b). Governance-Regime der Schulprofilierung. In H. Altrichter, M. Heinrich \& K. Soukup-Altrichter (Hrsg.), Schulentwicklung durch Schulprofilierung. Zur Veränderung von Koordinationsmechanismen im Schulsystem (S. 217-239). Wiesbaden: Springer VS.

Arbeitsgruppe „Lernen vor Ort“ (Hrsg.). (2016). Kommunales Bildungsmanagement als sozialer Prozess. Studien zu „Lernen vor Ort“. Wiesbaden: Springer VS.

Ball, S. J. (1993). Education markets, choice and social class: the market as a class strategy in the UK and the USA. British Journal of Sociology of Education, 14(1), 3-19.

Ball, S. J. (2003). Class strategies and the education market. The middle classes and social advantage. London/New York: RoutledgeFalmer.

Ball, S. J., Bowe, R., \& Gewirtz, S. (1996). School choice, social class and distinction: the realization of social advantage in education. Journal of Eduation Policy, 11(1), 89-112.

Baumert, J., Maaz, K., Gresch, C., McElvany, N., Anders, Y., Jonkmann, K., Neumann, M., \& Watermann, R. (2010). Der Übergang von der Grundschule in die weiterführende Schule - Leistungsgerechtigkeit und regionale, soziale und ethnisch-kulturelle Disparitäten: Zusammenfassung der zentralen Befunde. In K. Maaz, J. Baumert, C. Gresch \& N. McElvany (Hrsg.), Der Übergang von der Grundschule in die weiterführende Schule. Leistungsgerechtigkeit und regionale, soziale und ethnisch-kulturelle Disparitäten (S. 5-22). Bonn: BMBF.

Baur, C. (2013). Schule, Stadtteil, Bildungschancen. Wie ethnische und soziale Segregation Schüler/-innen mit Migrationshintergrund benachteiligt. Bielefeld: transcript.

Becker, R., \& Hadjar, A. (2011). Meritokratie - Zur gesellschaftlichen Legitimation ungleicher Bildungs-, Erwerbs- und Einkommenschancen in modernen Gesellschaften. In R. Becker (Hrsg.), Lehrbuch der Bildungssoziologie (2. Aufl. S. 37-62). Wiesbaden: Springer VS.

Bellin, N. (2009). Klassenkomposition, Migrationshintergrund und Leistung. Mehrebenenanalysen zum Sprach- und Leseverständnis von Grundschülern. Wiesbaden: VS.

Berends, M., Primus, A., \& Springer, M. G. (Hrsg.). (2019). Handbook of research on school choice. New York: Routledge.

Berkemeyer, N., \& Meißner, S. (2017). Soziale Ungleichheiten im Schulsystem und das Desiderat einer Soziologie der Schule. In M.S. Baader \& T. Freytag (Hrsg.), Bildung und Ungleichheit in Deutschland (S. 229-253). Wiesbaden: Springer VS.

Bloch, R., Breidenstein, G., Krüger, J.O., Mader, M., \& Winter, D. (2019). Die prekäre Legitimierung des limitierten Zugangs zu exklusiven Bildungseinrichtungen. In W. Helsper, H.-H. Krüger \& J. Lüdemann (Hrsg.), Exklusive Bildung und neue Ungleichheit. 65. Beiheft der Zeitschrift für Pädagogik (S. 267-276). Weinheim/Basel: Beltz Juventa.

Boterman, W., Musterd, S., Pacchi, C., \& Ranci, C. (2019). School segregation in contemporary cities: socio-spatial dynamics, institutional context and urban outcomes. Urban Studies, 56(15), 3055-3073.

Boudon, R. (1974). Education, opportunity, and social inequality. Changing prospects in western society. New York u. a.: John Wiley \& Sons.

Bourdieu, P., \& Passeron, J.-C. (1971). Die Illusion der Chancengleichheit. Untersuchungen zur Soziologie des Bildungswesens am Beispiel Frankreichs. Stuttgart: Klett.

Bourdieu, P., \& Passeron, J.-C. (1973). Grundlagen einer Theorie der symbolischen Gewalt. Frankfurt a. M.: Suhrkamp.

Bourdieu, P. (2010). Ortseffekte. In P. Bourdieu, et al. (Hrsg.), Das Elend der Welt (2. Aufl. S. 117-123). Konstanz: UVK.

Breidenstein, G., Krüger, J. O., \& Roch, A. (2020). Schulwahl als Elitebildung? Zur Bearbeitung des Segregationsverdachts im elterlichen Diskurs. In J. O. Krüger, A. Roch \& G. Breidenstein (Hrsg.), Szenarien der Grundschulwahl (S. 41-56). Wiesbaden: Springer VS.

Bremerich-Vos, A., Wendt, H., \& Hußmann, A. (2017). Bausteine adaptiven Leseunterrichts angesichts gewachsener Heterogenität. In A. Hußmann, et al. (Hrsg.), IGLU 2016. Lesekompetenzen von Grund- 
schulkindern in Deutschland im internationalen Vergleich (S. 297-314). Münster/New York: Waxmann.

Clausen, M. (2006). Warum wählen Sie genau diese Schule? Eine inhaltsanalytische Untersuchung elterlicher Begründungen der Wahl der Einzelschule innerhalb eines Bildungsgangs. Zeitschrift für Pädagogik, 52(1), 69-90.

Ditton, H. (2007). Schulwahlentscheidung unter sozial-regionalen Bedingungen. In O. Böhm-Kasper, C. Schuchart \& U. Schulzeck (Hrsg.), Kontexte von Bildung. Erweiterte Perspektiven in der Bildungsforschung (S. 21-38). Münster u. a.: Waxmann.

Ditton, H. (2013). Kontexteffekte und Bildungsungleichheit: Mechanismen und Erklärungsmuster. In R. Becker \& A. Schulze (Hrsg.), Bildungskontexte. Strukturelle Voraussetzungen und Ursachen ungleicher Bildungschancen (S. 173-206). Wiesbaden: Springer VS.

Ditton, H. (2014). Bildung und Region (NEPS Working Paper No. 44). Bamberg: Leibniz-Institut für Bildungsverläufe. Nationales Bildungspanel. https://www.neps-data.de/Portals/0/Working\%20Papers/ WP_XLIV.pdf. Zugegriffen: 20. Okt. 2019.

Ditton, H., \& Krüsken, J. (2007). Sozialräumliche Segregation und schulische Entwicklung. Diskurs Kindheits- und Jugendforschung, 2(1), 23-38.

Dumont, H., Maaz, K., Neumann, M., \& Becker, M. (2014). Soziale Ungleichheiten beim Übergang von der Grundschule in die Sekundarstufe I: Theorie, Forschungsstand, Interventions- und Fördermöglichkeiten. Zeitschrift für Erziehungswissenschaft, 24(Sonderheft), 141-166.

Dumont, H., Neumann, M., Maaz, K., \& Trautwein, U. (2013). Die Zusammensetzung der Schülerschaft als Einflussfaktor für Schulleistungen. Psychologie in Erziehung und Unterricht, 60, 163-183.

Farwick, A. (2012). Segregation. In F. Eckardt (Hrsg.), Handbuch Stadtsoziologie (S. 381-419). Wiesbaden: Springer VS.

Fincke, G., \& Lange, S. (2012). Segregation an Grundschulen: Der Einfluss der elterlichen Schulwahl. Berlin: Sachverständigenrat deutscher Stiftungen für Integration und Migration. https://www.svrmigration.de/wp-content/uploads/2014/11/Segregation_an_Grundschulen_SVR-FB_WEB.pdf. Zugegriffen: 20. Okt. 2019.

Friedrichs, J. (2011). Ethnische und soziale Segregation in deutschen Großstädten. In W. Hanesch (Hrsg.), Die Zukunft der „Sozialen Stadt“ (S. 49-61). Wiesbaden: VS.

Geißler, R. (2005). Die Metamorphose der Arbeitertochter zum Migrantensohn. Zum Wandel der Chancenstruktur im Bildungssystem nach Schicht, Geschlecht, Ethnie und deren Verknüpfungen. In P. A. Berger \& H. Kahlert (Hrsg.), Institutionalisierte Ungleichheiten. Wie das Bildungswesen Chancen blockiert (S. 71-100). Weinheim: Juventa.

Habeck, C., Schwarz, J., Gruehn, S., \& Koinzer, T. (2017). Public and private school choice in the German primary education sector: an empirical analysis of parental reasons. In T. Koinzer, R. Nikolai \& F. Waldow (Hrsg.), Private schools and school choice in compulsory education (S. 201-221). Wiesbaden: Springer VS.

Hauf, T. (2007). Innerstädtische Bildungsdisparitäten an der Übergangsschwelle von den Grundschulen zum Sekundarschulsystem. Zeitschrift für Pädagogik, 53(3), 299-313.

Häußermann, H., \& Kronauer, M. (2009). Räumliche Segregation und innerstädtisches Ghetto. In R. Stichweh \& P. Windolf (Hrsg.), Inklusion und Exklusion: Analysen zur Sozialstruktur und sozialen Ungleichheit (S. 157-173). Wiesbaden: VS.

Heinzel, F., \& Parade, R. (2020). Zum (prekären) Selbstverständnis der Grundschule als „Schule für alle Kinder“. In N. Skorsetz, M. Bonanati \& D. Kucharz (Hrsg.), Diversität und soziale Ungleichheit. Herausforderung an die Integrationsleistung der Grundschule (S. 41-45). Wiesbaden: Springer VS.

Helbig, M. (2010). Neighborhood does matter! Soziostrukturelle Nachbarschaftscharakteristika und Bildungserfolg. Kölner Zeitschrift für Soziologie und Sozialpsychologie, 62, 655-679.

Helbig, M., \& Jähnen, S. (2018). Wie brüchig ist die soziale Architektur unserer Städte? Trends und Analysen der Segregation in 74 deutschen Städten. Discussion Paper P-2018-001. Wissenschaftszentrum Berlin für Sozialforschung. https://bibliothek.wzb.eu/pdf/2018/p18-001.pdf. Zugegriffen: 6. Jan. 2020.

Helbig, M., \& Nikolai, R. (2019). Bekommen die sozial benachteiligsten Schüler*innen die „,besten“ Schulen? Eine explorative Studie über den Zusammenhang von Schulqualität und sozialer Zusammensetzung von Schulen am Beispiel Berlins. https://bibliothek.wzb.eu/pdf/2019/p19-002.pdf. Zugegriffen: 6. Jan. 2020. Discussion Paper P-2019-002. Wissenschaftszentrum Berlin für Sozialforschung.

Helsper, W., Kramer, R.-T., \& Thiersch, S. (Hrsg.). (2014). Schülerhabitus. Theoretische und empirische Analysen zum Bourdieuschen Theorem der kulturellen Passung. Wiesbaden: Springer VS.

Helsper, W., Krüger, H.-H., Bloch, R., \& Mitterle, A. (2019). Horizontale und vertikale Differenzierungsprozesse im deutschen Bildungssystem. Neue Formen sozialer Stratifikation als Ausdruck von Elite- 
bildung? In W. Helsper, H.-H. Krüger \& J. Lüdemann (Hrsg.), Exklusive Bildung und neue Ungleichheit. Ergebnisse der DFG-Forschergruppe „Mechanismen der Elitebildung im deutschen Bildungssystem “. 65. Beiheft der Zeitschrift für Pädagogik (S. 252-266). Weinheim/Basel: Beltz Juventa.

Hopf, W. (2017). Von der Gleichheit der Bildungschancen zur Bildungsgerechtigkeit für alle - ein Abschied auf Raten vom Gleichheitsideal. In M.S. Baader \& T. Freytag (Hrsg.), Bildung und Ungleichheit in Deutschland (S. 23-37). Wiesbaden: Springer VS.

Horr, A. (2016). Nachbarschaftseffekte. In C. Diehl, C. Hunkler \& C. Kristen (Hrsg.), Ethnische Ungleichheiten im Bildungsverlauf (S. 397-430). Wiesbaden: Springer VS.

Hummrich, M. (2015). Schule und Sozialraum. Erziehungswissenschaftliche Perspektiven. In A. ElMafaalani, S. Kurtenbach \& K.P. Strohmeier (Hrsg.), Auf die Adresse kommt es an... Segregierte Stadtteile als Problem- und Möglichkeitsräume begreifen (S. 168-187). Weinheim/Basel: Beltz Juventa.

Jurczok, A., \& Lauterbach, W. (2014). Schulwahl von Eltern: Zur Geografie von Bildungschancen in benachteiligten städtischen Bildungsräumen. In P. A. Berger, et al. (Hrsg.), Urbane Ungleichheiten (S. 135-155). Wiesbaden: Springer VS.

Jurzczok, A. (2019). Schulwahl unter ,gleichwertigen“ Einzelschulen. Elterliche Übergangsentscheidungen im zweigliedrigen Sekundarschulsystem. Wiesbaden: Springer VS.

Kahlert, H. (2008). Bildung und Erziehung im Übergang zur Wissensgesellschaft. In H. Willems (Hrsg.), Lehr(er)buch Soziologie (Bd. 2, S. 773-796). Wiesbaden: VS.

Kessl, F., \& Reutlinger, C. (2019). Vorwort. In F. Kessl \& C. Reutlinger (Hrsg.), Handbuch Sozialraum. Grundlagen für den Bildungs- und Sozialbereich 2. Aufl. Wiesbaden: Springer VS.

Koch, K. (2008). Von der Grundschule zur Sekundarstufe. In W. Helsper \& J. Böhme (Hrsg.), Handbuch der Schulforschung (2. Aufl. S. 577-592). Wiesbaden: Springer VS.

Koinzer, T., \& Leschinsky, A. (2009). Privatschulen in Deutschland. Zeitschrift für Pädagogik, 55(5), $669-685$.

Kramer, R.-T. (2011). Abschied von Bourdieu? Perspektiven ungleichheitsbezogener Bildungsforschung. Wiesbaden: VS.

Krüger, J. O. (2020). Die schulische Elternwahl. Auswahl an Grundschulen in privater Trägerschaft. In J. O. Krüger, A. Roch \& G. Breidenstein (Hrsg.), Szenarien der Grundschulwahl (S. 57-70). Wiesbaden: Springer VS.

Krüger, J. O., Breidenstein, G., Tyagunova, T., \& Böckelmann, K. (2015). Von Schullandschaften zu Schulmärkten? Ambivalenzen im Diskurs zur Grundschulwahl. Zeitschrift für Grundschulforschung, 8(2), 42-54.

Lintorf, K., Guill, K., \& Wendt, H. (2017). „Was dein Kind kann, kann meins schon lange!“ Effekte der sozialen Komposition von Grundschulklassen auf den Übergang. Zeitschrift für Erziehungswissenschaft, 20(4), 711-727.

Makles, A.M., \& Schneider, K. (2012). Freie Wahl der Grundschule: Wie entscheiden sich Eltern und welche Konsequenzen hat die Schulwahl für die Segregation? Die Deutsche Schule, 104(4), 332-346.

Makles, A. M., Schneider, K., \& Terlinden, B. (2019). Schulische Segregation und Schulwahl. Eine Analyse mit den Daten der Bremer Schülerindividualstatistik. In D. Fickermann \& W. Weishaupt (Hrsg.), Bildungsforschung mit Daten der amtlichen Statistik (S. 176-196). Münster/New York: Waxmann.

Mayer, T. (2017). School choice and the urban neighbourhood: segregation processes in the German primary sector with special reference to private schools. In T. Koinzer, R. Nikolai \& F. Waldow (Hrsg.), Private schools and school choice in compulsory education (S. 153-175). Wiesbaden: Springer VS.

Mayer, T., \& Koinzer, T. (2019). Schulwahl und Bildungsungleichheit - Ein holistisches Modell zur Erklärung von Segregation und Bildungsdisparitäten bei der Einzelschulwahl. Pädagogische Rundschau, 73(3), 265-279.

Morris-Lange, S., Wendt, H., \& Wohlfahrth, C. (2013). Segregation an deutschen Schulen. Ausmaß, Folgen und Handlungsempfehlungen für bessere Bildungschancen. Berlin: Sachverständigenrat deutscher Stiftungen für Integration und Migration. https:/www.svr-migration.de/wp-content/uploads/ 2013/07/SVR-FB_Studie-Bildungssegregation_Web.pdf. Zugegriffen: 8. Okt. 2019.

Neumann, M., Milek, A., Maaz, K., \& Gresch, C. (2010). Zum Einfluss der Klassenzusammensetzung auf den Übergang von der Grundschule in die weiterführende Schule. In K. Maaz, J. Baumert, C. Gresch \& N. McElvany (Hrsg.), Der Übergang von der Grundschule in die weiterführende Schule. Bonn/ Berlin: BMBF.

Nonnenmacher, A. (2015). Städtische Armutsquartiere und Bildung. In T. Coelen, A. J. Heinrich \& A. Million (Hrsg.), Stadtbaustein Bildung (S. 137-145). Wiesbaden: Springer VS.

Noreisch, K. (2007). School catchment area evasion: the case of Berlin. Germany. Journal of Education Policy, 22(1), 69-90. 
OECD (2018). Erfolgsfaktor Resilienz. Warum manche Jugendliche trotz schwieriger Startbedingungen in der Schule erfolgreich sind - und wie Schulerfolg auch bei allen anderen Schülerinnen und Schülern gefördert werden kann. http://www.oecd.org/berlin/publikationen/VSD_OECD_Erfolgsfaktor \%20Resilienz.pdf. Zugegriffen: 29. Jan. 2019. Eine PISA-Sonderauswertung der Organisation für wirtschaftliche Zusammenarbeit und Entwicklung (OECD) in Kooperation mit der Vodafone Stiftung Deutschland.

Radtke, F.-O. (2007). Segregation im deutschen Schulsystem. In W.-D. Bukow, C. Nikodem, E. Schulze \& E. Yildiz (Hrsg.), Was heißt hier Parallelgesellschaft? Zum Umgang mit Differenzen (S. 201-212). Wiesbaden: VS.

Riedel, A., Schneider, K., Schuchart, C., \& Weishaupt, H. (2010). School choice in German primary schools: How binding are school districts? Journal for Educational Research Online, 2, 94-120.

Roch, A., Breidenstein, G., \& Krüger, J.O. (2019). Die Chiffre des ,Bildungsinteresses‘ im Diskurs der Grundschulewahl. In W. Helsper, H.-H. Krüger \& J. Lüdemann (Hrsg.), Exklusive Bildung und neue Ungleichheit. 65. Beiheft der Zeitschrift für Pädagogik (S. 107-123). Weinheim/Basel: Beltz Juventa.

Rohde, L. A., Campani, F., Oliveira, J.R. G., Rohde, C. W., Rocha, T., \& Ramal, A. (2019). Parental reasons for school choice in elementary school: a systematic review. Journal of School Choice, 13(3), 287-304.

Schräpler, J.-P., \& Weishaupt, H. (2019). Grundschule und sozialräumliche Ungleichheit. Zeitschrift für Grundschulforschung, 12(2), 415-437.

Schuchart, C., Schneider, K., Weishaupt, H., \& Riedel, A. (2011). Welchen Einfluss hat die Wohnumgebung auf die Grundschulwahl der Eltern? - Analysen zur Bedeutung von kontextuellen und familiären Merkmalen auf das Wahlverhalten. Schumpeter Discussion Papers der Bergischen Universität Wuppertal. http://elpub.bib.uni-wuppertal.de/edocs/dokumente/fbb/wirtschaftswissenschaft/ sdp/sdp11/sdp11009.pdf. Zugegriffen: 20. Okt. 2019.

Schwarz, J., Habeck, C., Gruehn, S., \& Koinzer, T. (2018). „Welche Schule passt zu meinem Kind?“ Elterliche Schulwahlentscheidungen und die Frage der Passung im öffentlichen und privaten Grundschulwesen. Zeitschrift für Pädagogik, 64(5), 612-634.

Steinmeier, F.-W. (2019in). 100 Jahre Grundschule. Rede beim Festakt „100 Jahre Grundschule“ zur Eröffnung des Bundesgrundschulkongresses „Kinder Lernen Zukunft“ am 13. September 2019 in Frankfurt am Main. https:/www.bundespraesident.de/SharedDocs/Downloads/DE/Reden/2019/ 09/190913-Grundschule-100-Jahre.pdf; jsessionid=A22A4953229C1AA358B1606F22947218.2_ cid362?_blob=publicationFile. Zugegriffen: 30. Okt. 2019.

Stirner, P., Hoffmann, L., Mayer, T., \& Koinzer, T. (2019). Eine gemeinsame Grundschule für alle? Die Grundschule als Ort sozio-ökonomischer Ungleichheit und Segregation. Zeitschrift für Grundschulforschung, 12(2), 439-455.

Stošić, P. (2015). Horizontale Segregation im deutschen Schulsystem. In L. Fölker, T. Hertel \& N. Pfaff (Hrsg.), Brennpunkt (-) Schule. Zum Verhältnis von Schule, Bildung und urbaner Segregation (S. 29-48). Opladen: Barbara Budrich.

Terpoorten, T. (2014). Räumliche Konfiguration der Bildungschancen. Segregation und Bildungsdisparitäten am Übergang in die weiterführenden Schulen im Agglomerationsraum Ruhrgebiet. ZEFIRMaterialien Band 3. Bochum: Zefir. http://www.zefir.rub.de/mam/content/zefir_materialien_3_r\%C3 \%A4umliche_konfiguration_der_bildungschancen_2014.pdf. Zugegriffen: 20. Okt. 2019.

Ullrich, H., \& Strunck, S. (2012). Private Schulen in Deutschland - Entwicklungen und Diskurse. In H. Ullrich \& S. Strunck (Hrsg.), Private Schulen in Deutschland. Entwicklungen, Profile, Kontroversen (S. 11-25). Wiesbaden: VS.

Van Ewijk, R., \& Sleegers, P. (2010). The effect of peer socioeconomic status on student achievement: a metaanalysis. Educational Research Review, 5(2), 134-150.

Vester, M. (2006). Die ständische Kanalisierung der Bildungschancen. Bildung und soziale Ungleichheit zwischen Boudon und Bourdieu. In W. Georg (Hrsg.), Soziale Ungleichheit im Bildungssystem. Eine empirisch-theoretische Bestandsaufnahme (S. 13-54). Konstanz: UVK.

Wehrheim, J. (2016). Raum. In J. Kopp \& A. Steinbach (Hrsg.), Grundbegriffe der Soziologie (S. 277-280). Wiesbaden: Springer VS. 